\title{
Parasite Vaccines: Recent Progress in, and Problems Associated with their Development
}

\author{
D.P. Knox \\ Moredun Research Institute, International Research Centre, Pentlands Science Park, Bush Loan, Penicuik, Midlothian \\ EH26 OPZ, Scotland, UK
}

\begin{abstract}
Metazoan and protozoan parasites are major causes of human and animal disease causing extensive morbidity and mortality, particularly in tropical and sub-tropical climatic regions. WHO estimates that one person in every four is affected by parasitic worms with disease outcomes ranging from chronic symptoms, blindness, disfiguration to death. In addition, gastrointestinal parasitism is one of the greatest animal health constraints worldwide, both to commercial and subsistence farmers. While substantial progress has been made in identifying potential protective antigens, vaccine development is impaired because it is difficult, often impossible, to cultivate many of the major target parasites in vitro, most parasites present different developmental stages to the host immune system, most show considerable antigenic diversity and there is growing evidence that they directly modulate the host immune system to their advantage. While progress has been made in the last decade in the cloning and expression of protective antigens from a large number of parasites, the majority have failed to stimulate practically useful levels of protective immunity in trials. This review is intended to provide the reader with an overview of the nature of the disease problem being addressed using selected examples and the approaches being taken to develop vaccines to counter the adverse effects of infection. It is not intended to be fully comprehensive and the interested reader can access more in depth discussion from cited references and using on-line search resources.
\end{abstract}

Keywords: Parasite, vaccination, recombinant, sub-unit vaccine.

\section{THE GLOBAL IMPACT OF PARASITISM}

Limiting the impact of parasitism in both man and livestock relies almost exclusively on the use of antiparasitic drugs. Control is threatened by the widespread appearance of drug-resistant parasites in animals [1] and man [2-4]. There is an urgent need to develop novel, sustainable control procedures with vaccination to the fore.

The ability to produce recombinant parasite proteins in the early 1980's (e.g. [5, 6]) was heralded as a major breakthrough for vaccine development yet, 25 years on, only a few recombinant vaccines against parasitic diseases of livestock have reached the point of being marketed. The first recombinant vaccine against a human parasite continues to remain elusive.

The prevalence of the major diseases in man is summarised in Table $\mathbf{1}$ above. Intestinal and protozoan infections are the most common parasitic diseases with, according to WHO, 3.5 billion people affected and 450 million, mainly children, ill as a result. Co-infection with different parasites is common, and the illness is often made worse by co-existing malnutrition. Prevalence is predicted to increase as it is anticipated that, by $2050,>50 \%$ of people in the developing world will be living in urban and peri-urban conditions, many of them in shanty towns, where several

*Address correspondence to this author at the Moredun Research Institute, International Research Centre, Pentlands Science Park, Bush Loan, Penicuik, Midlothian EH26 0PZ, Scotland, UK; Tel: +44 (0)131 445 5111; Fax: +44 (0)131 445 6111; E-mail: Knoxd@mri.sari.ac.uk parasites are readily transmitted [7]. Parasitic diseases also have a major impact on livestock production worldwide with infection arising from a range of helminth, protozoan and ecto-parasites. Amongst the most important is the pathogenic, blood-feeding nematode parasite of sheep and goats Haemonchus contortus. This parasite is particularly prevalent in tropical and sub-tropical regions and infection and its intensity has direct consequences for both the large scale sheep/goat producer and, perhaps of more importance, the resource poor small holder where production directly affects family diet and, ultimately, survival.

Table 1. The Major Parasitic Diseases Affecting Man (Adapted from [7])

\begin{tabular}{|c|c|c|c|}
\hline Disease & $\begin{array}{c}\text { Population at Risk } \\
\left(\times \mathbf{1 0}^{\mathbf{6}}\right)\end{array}$ & $\begin{array}{c}\text { Cases } \\
\left(\times \mathbf{1 0}^{\mathbf{6}}\right)\end{array}$ & $\begin{array}{c}\text { Mortality } \\
\left(\times \mathbf{1 0}^{\mathbf{3}}\right)\end{array}$ \\
\hline \hline Malaria & $>2100$ & $270-400$ & 1120 \\
\hline African trypanosomiasis & $>60$ & $0.3-0.5$ & 49 \\
\hline Chagas disease & 120 & 17 & 13 \\
\hline Leishmaniasis & 350 & 12 & 57 \\
\hline Schistosomiasis & 600 & $>200$ & 15 \\
\hline Onchocerciasis & 120 & 18 & 0 \\
\hline Lymphatic filariasis & 1000 & 120 & 0 \\
\hline Intestinal protozoa & 3500 & 450 & 65 \\
\hline Geohelminths & 4500 & $\sim 3000$ & 17 \\
\hline
\end{tabular}




\section{ZOONOTIC ASPECTS OF PARASITISM}

Many parasite diseases are zoonoses with disease arising from both metazoan and protozoan agents. Hydatid disease, due to infection with tapeworms of the Echinococcus genera, is a public health problem in many parts of the world. The tapeworm lives in the intestines of dogs and other canids and humans become affected if they ingest the eggs of the organism, the disease being a sanitation issue. Disease caused by the digenetic trematode $\mathrm{S}$. japonicum is a major zoonosis in South East Asia affecting over 250 million people and causing a quarter of a million deaths annually in sub-Saharan Africa alone [8]. It affects more than 40 million people in the tropical and sub-tropical zones of China [9, 10]. Fascioliosis is caused by infection with the liver fluke, Fasciola hepatica. Widespread infestation is common in sheep and bovines, and it can also occur in humans. Infection in humans arises from ingestion of uncooked, unwashed aquatic vegetables contaminated with encysted larvae. While animals can carry large worm burdens without developing serious disease, Fasciola spp. can cause severe, even fatal disease in humans [11-13] Human infection is always associated with local endemic animal fasciolosis [10]. The protozoan parasite, Giardia, is found worldwide and causes significant gastrointestinal disease in dogs, cats, and people. Cryptosporidium parvum causes severe gastrointestinal tract disease with infection of immunosuppressed individuals particularly severe.

\section{CURRENT CONTROL}

Drugs remain central to alleviating clinical disease and for larger scale disease control programmes. However, available drugs have often been in use for decades and drug resistance in the target parasites is now prevalent and, particularly in the case of livestock, threatening sustainable control. The issues surrounding drug resistance in the major human parasites is extensively discussed in Trends in Parasitology (2003) 19, Issue 11. In livestock, drug resistance has been reported to every anthelmintic class in every livestock host [1]. In some regions, multi-drug resistant nematodes are becoming prevalent and threaten the viability of small-ruminant production units. A review of this nature must also draw attention to the scourge of ectoparasites with tick and mite infestations prevalent in man and animals.

For some parasitic diseases prevalence should eventually decrease with improved health education, water supply and sanitation. For vector-borne diseases this is not necessarily so. In many areas, malaria is out of control and there is increasing drug resistance, particularly in sub-Saharan Africa. It affects all socio-economic groups. The only practical control measures are protection against mosquitoes, such as with insecticideimpregnated bed-nets. Epidemic leishmaniasis and trypanosomiasis affect the most vulnerable members of society unable to protect themselves. Major efforts coordinated by WHO, other international organizations and philanthropic charity activity and are in the process of seeking novel methods to control if not eradicate several of the major parasites of man and animals.

\section{VACCINE EFFICACY}

A question which needs to be addressed early in a vaccine development project is how good does the vaccine have to be? In the case of a parasite vaccine, the level of protection required will vary depending on whether the vaccine is a stand alone control procedure or is applied in conjunction with management procedures which may reduce exposure to the parasite. Minimising or eliminating the clinical consequences of infection should be a goal [14-19]. In general terms, the "performance" requirements for a vaccine are defined on the basis of epidemiological data and mathematical modelling [14]. However, user perception is likely to be very influential. For example, livestock producers are likely to compare a vaccine with control achieved with anthelmintic drugs and ectoparasiticides, which, when first introduced, approach $100 \%$ efficacy. However, computer modeling of population dynamics suggest this level of efficacy is not required to achieve practical control $[20,21]$. Therefore, the introduction of a vaccine is going to require a sustained educational effort.

\section{THE PROTECTIVE IMMUNE RESPONSE}

Vaccine development has been hampered by a lack of definition of the precise immune effectors of parasite attrition and the antigens which stimulate them. Wynn \& Hoffmann [22] noted that successful vaccine development for schistosomiasis had been hindered by a lack of consensus on the type of immune response required and an incomplete knowledge of the effectors mechanisms which mediate immunity. The maintenance of natural immunity is often dependent on repeated infection, may be stage-specific and will be dependent on different antibody classes and T-cell responses. Despite it being almost 30 years since the technology to produce recombinant proteins became available, recombinant proteins with the required efficacy are rare, spectacular exceptions being vaccine developments in ticks and cestodes, these developments constituting land mark achievements [23, 24].

\section{PROGRESS TOWARDS SUB-UNIT VACCINES - SOME EXAMPLES}

Vaccine development originally focused on a fractionate and vaccinate approach. Antigens are purified from parasite extracts or are harvested following in vitro culture using a variety of protein fractionation procedures [25] and then evaluated for protective efficacy in control trials in the target host or, frequently, in a laboratory infection model. Antigens can be selected on the basis of presumed functional importance to parasite survival such as enzymes required for feeding/migration, immunomodulatory molecules or on the basis of immune recognition by hosts rendered immune to infection by repeated exposure. In the case of helminth infection, ES in vitro released proteins (often termed excretory/secretory [ES]) have been prime targets because they are readily accessible to the host immune response, are immunogenic and many are functionally important. The examples described below highlight some real successes and, in most instances, painstaking and often frustrating work towards vaccine development. Moreover, space would not permit a comprehensive description of all the antigens being targeted in individual parasites so, again, the author has been selective.

\section{CESTODES}

A review of this nature can only start with a real success story and ground-breaking progress has been made in 
developing cestode vaccines. Man is afflicted by a number of different cestode parasites including the pork tapeworm Taenia solium, the beef tapeworm, T. saginata and the hydatids Echinococcus granulosus and E. multilocularis. T. solium and $T$. saginata have a worldwide distribution but incidence is considerably higher in developing countries. The tapeworm larval cyst, containing the infective onchosphere stage, is ingested with poorly cooked infected meat. Adults establish in te intestines. The adult may live as long as 25 years and pass gravid proglottids, containing eggs, with the faeces. The eggs can persist on vegetation for several days and are consumed by cattle or pigs in which they hatch and form cysticerci. The eggs can also infect humans and cause cysticercosis where cysts can establish in the lungs, liver, eye and brain resulting in blindness and neurological disorders. Gastrointestinal symptoms arise from the presence of the adult tape worm while cysticercosis symptoms are due to the host inflammatory/immune responses to the parasite and the abnormal mechanical presence of the cyst.

Recombinant antigen vaccines targeting onchosphere proteins [24] have been developed against infection with a number of Taenia species including Taenia ovis in sheep [26, 27], Taenia saginata in cattle [28] and Taenia solium in pigs $[29,30]$. These vaccines are based on groups of oncosphere proteins designated $16 \mathrm{k}, 18 \mathrm{k}$ and $45 \mathrm{~W}$ groups, after their original description in T. ovis [26]. Protection induced by vaccination approaches $100 \%$. The $45 \mathrm{~W}$ vaccine against Taenia ovis infection in sheep [26] was the first highly effective recombinant vaccine against a parasitic infection. A similar approach using immunoblotting and in vitro onchosphere killing identified a putative protective molecule (EG95) in Echinococcus granulosus [31]. Despite these impressive successes, these vaccines have yet to be applied due to commercial, economic and socio-economics reasons [24]. However, it cannot be overemphasized that this work, and the work immediately below on ticks, is groundbreaking providing proof of concept for the development of sub-unit vaccines against complex metazoan parasites.

\section{ECTOPARASITES}

Continuing the success theme, a recombinant antigen vaccine against the blood-feeding cattle tick was successfully developed in Australia. Naturally acquired immunity to a range of tick species in a variety of hosts was used to monitor antigen recognition but this approach failed to identify any useful protective antigens. However, the fractionate and vaccinate approach was successful, a series of protein fractions from the blood-feeding cattle tick Boophilus microplus being tested in vaccine trials which led to the discovery of the highly protective Bm86 antigen [32, 33]. This antigen is found on the microvilli of the tick gut digest cells [34]. Once ingested by feeding ticks, antibody to Bm86 leads to lysis of the digest cells that line the tick gut, a process which may be complement-mediated. Bm86 is expressed in the tick gut and is not recognized by antibody arising from natural infestation. Antigens with this property have been termed "hidden" or "concealed" and immunity stimulated referred to as "artificial". The process of developing a commercial vaccine from this discovery has been briefly described [35].

\section{HAEMONCHUS CONTORTUS IN SHEEP}

Gut membrane proteins have also been effectively employed as vaccine antigens for the control of the bloodfeeding nematode of sheep and goats $H$. contortus [36] and are offering promise as vaccine components against hookworms [37]. High levels of protective immunity, evidenced by reductions in worm burdens and egg output of $>90 \%$, can be achieved against Haemonchus by targeting proteins expressed on the intestinal microvilli of the blood feeding fourth larval and adult stages [38]. Several have been ascribed roles in blood meal digestion including an aminopeptidase (H11, reviewed in [39]) and a protein complex termed H-gal-GP which contains aspartyl- and metallo-proteases [40-42]. Vaccination of dogs with a cysteine haemoglobinase, Ac-CP-2 [43] or an aspartyl protease (Ac-APR-1, [44] provided proof that haemoglobinolytic proteases expressed in recombinant form are potential efficacious vaccines against hookworm infections. Haemoglobin digestion is a multi-enzyme synergistic cascade in blood-feeding parasites $[45,46]$ and some of the enzymes involved in this process have been identified in hookworms and Haemonchus [47]. Bacterially expressed recombinant versions of cysteine protease expressed in the intestine of adult Haemonchus contortus have shown moderate efficacy in sheep.

\section{SCHISTOSOMIASIS}

Schistosomiasisis affects an estimated 300 million people worldwide. The causative agents are snail-transmitted, water-borne parasitic helminths. The schistosomes have complex life cycles using two separate hosts to complete their development. Severe consequences of infection include bladder cancer or renal failure (Schistosoma haematobium) and liver fibrosis and portal hypertension (S. mansoni). Again, control is largely dependent on mass chemotherapy but resistance is a concern. Disease is endemic in over 70 countries causing tens of thousands of deaths. S. haematobium is found in the Middle East and Africa and Southern Europe and affects the bladder causing urinary schostosomiasis. S. mansoni, is present in most African countries north of the equator as well as parts of Central and South America and causes intestinal schostosomiasis. S. japonicum is endemic in China where bovines are the main reservoir, as well as in Indonesia and the Philippines (with dogs and pigs as reservoir). S. bovis is an important parasite affecting cattle, as well as sheep and goats and is prevalent in South East Asia.

Irradiated larval vaccines were a focus of study in the 1970 s with several early studies being conducted in sheep challenged with S. mattheei and S. bovis. Vaccination of mice with radiation-attenuated cercariae reduced worm burdens by $90 \%$ compared to controls and this vaccine model has been exploited to identify the antigens responsible for protection. The approaches being used for vaccine development have been extensively reviewed [48]. Lead antigens include several proteins expressed on the surface of the adult stage with particular current interest in transmembrane proteins. Tetraspanins are fourtransmembrane-domain proteins found on the surfaces of eukaryotic cells, including B and T cells and at least three of these show promise as vaccines [49]. $\mathrm{Sm} 23$ is one of the 
independently tested WHO/TDR vaccine candidates and is a tetraspanin expressed in the tegument of $S$. mansoni. Sm23 is not effective when given as a recombinant protein with alum but is effective when delivered as a DNA vaccine [50]. Recently, two new S. mansoni tetraspanins (SmTSP-1 and SmTSP-2) were identified [49]. Recombinant TSP-2, but not TSP-1, is strongly recognized by IgG1 and IgG3 (but not $\mathrm{IgE}$ ) from naturally resistant individuals. It is not recognized by IgG from chronically infected or unexposed individuals. Both proteins induced good levels of protection $(>50 \%$ reductions in liver egg burdens) in immunized mice given an $S$. mansoni challenge. The authors noted that TSP-2 in particular provided protection in excess of the $40 \%$ benchmark set by the World Health Organization for progression of schistosome vaccine antigens into clinical trials.

Other antigens include a $97 \mathrm{kDa}$ paramyosin [51] and a $28 \mathrm{kDa}$ triose phosphate isomerase (TPI; eg [52]), the latter being ubiquitous in each stage. Given that the $S$. mansoni and S. japonicum sequences are very similar, trials of vaccine efficacy have focused on the latter with the aim of blocking parasite transmission [52]. Pigs vaccinated with a TPI DNA vaccine alone showed reductions in adult worm burdens of $48 \%$ with female parasites being more susceptible [53]. Vaccination reduced liver egg numbers and reduced granuloma size. Moreover, protective immunity was stimulated in water buffalo by vaccination with a DNA plasmid comprising a fusion of S. japonicum TPI, a heat shock protein and IL-12 [54]. McManus and Loukas [48] noted that the data were encouraging but that extensive triails were still needed to determine if a vaccine of this nature would reduce transmission as evidenced by reduced adult worm burdens, egg outputs and reductions in hepatic egginduced pathology.

There has been much focus on 26 and $28 \mathrm{kD}$ glutathioneS-transferases (GSTs), GSTs being thought to have a detoxification role for the parasite. Sm28-GST is expressed in the sub-tegumental tissues of the parasite and vaccination studies in rats and hamsters with the S. haematobium equivalent [55] indicated its significant protective potential. Trials in primates demonstrated an anti-fecundity effect [56]. Clinical testing of Sh28-GST in people showed that the vaccine was immunogenic and antibody inhibited the enzymatic activity of the recombinant protein [57].

A concerted effort, based in Egypt and supported by USAID, the Schistosomiasis Vaccine Development Programme (SVDP), is examining two S. mansoni antigens: paramyosin and a synthetic peptide construct containing multiple antigen epitopes (MAP) from the triose phosphate isomerase. Another vaccine candidate is a $14 \mathrm{kD}$ fatty acidbinding $S$. mansoni protein (Sm14) which provided 67\% protection against challenge with $S$. mansoni cercariae [58]. Sm14 is a cytosolic protein expressed in the basal lamella of the tegument and the gut epithelium [59] and is an attractive vaccine candidate because fatty acid binding proteins play a key role in schistosome nutrient acquisition.

McManus and Loukas (2008) [48] commented that an apparent efficacy ceiling of 40 to $50 \%$ was a roadblock to success. This is compounded by difficulties in obtaining good expression levels and in scaling up production according to good laboratory practice/GMP standards. More effective antigens may be selected by mining the publicly available $S$. mansoni and $\mathrm{S}$. japonicum transcriptomes in combination with DNA microarray profiling, proteomics, glycomics, and immunomics. The development of RNA interference to disrupt gene function is a big step forward and will enhance the ability to determine the functions of schistosome genes/proteins and it is hoped the techniques will helo define those essential for survival and reproduction. Silencing the expression of numerous $S$. mansoni genes has resulted in phenotypic changes, highlighting their importance as targets for vaccines and new drugs [60].

\section{THE HOOKWORMS}

The major human soil transmitted helminths (STH), Ascaris lumbricoides, Trichuris trichiura and the hookworms occur in 1221 million, 795 million, and 740 million people, respectively [61], and are among the most common pathogens of humans in developing countries. There is considerable epidemiological overlap among the STH infections and Disability-Adjusted Life Year (DALY) estimates indicate that the disease burden from human STH infections is nearly equivalent to better-known conditions such as malaria and tuberculosis [62]. They have broadranging effects on pregnancy, childhood growth, nutrition, and cognitive and intellectual development [63].

Human hookworm infection is caused by the nematode parasites Necator americanus and Ancylostoma duodenale and infection is a leading cause of anaemia and protein malnutrition, afflicting an estimated 740 million people in the developing nations of the tropics. The largest numbers of cases occur in impoverished rural areas of sub-Saharan Africa, Latin America, South-East Asia and China. N. americanus is the most common hookworm worldwide, while A. duodenale is more geographically restricted.

Hookworm infection arises by skin contact with infective third-stage larvae (L3). Adult hookworms are blood feeders and attach to the mucosa of the small intestine and cause intestinal blood loss. Typically, symptoms can arise from infections with as little as 50 to 150 of adult parasites. Infection induces an iron-deficiency anaemia which is in direct correlation with the number of parasites (as measured by quantitative egg counts). In children, chronic hookworm infection impairs physical and intellectual development, reduces school performance and attendance, and adversely affects future productivity and wage-earning potential [63].

Control is achieved by population-wide treatment with benzimidazole-based anthelmintics albendazole and the 2001 World Health Assembly advocated the anthelminthic treatment of $75 \%$ of all at-risk school-aged children by 2010 . However, drug treatment does not prevent reinfection and the frequent use of drugs required to maintain control would be likely to lead to drug resistance. Therefore, there is a drive to develop a safe and cost-effective vaccine.

The selection of the leading hookworm vaccine candidate, the Ancylostoma-secreted protein, ASP-2, was based on three major lines of evidence [64]. First, human anti-ASP-2 antibody responses are associated with a considerably reduced risk of acquiring heavy hookworm infection [65]. Second, when recombinant ASP-2 (expressed either in yeast or baculovirus) was used as vaccine in animal models, it resulted in reductions in host hookworm burden, 
hookworm size and fecundity [65-67]. Third, anti-ASP-2 antibody inhibits larval penetration through host tissue in vitro [65] suggesting that the vaccine elicits an antibody response that interferes with passage of larvae through host tissue. Of the L3 ES products tested, ASP-2 provided the greatest levels of protection in canines [65] and hamster models of hookworm infection and was, therefore, given the highest priority. The Na-ASP-2 vaccine is well-tolerated and induces a prolonged immune response in adults not exposed to hookworm, justifying further testing of this vaccine in an endemic [66, 68].

The most consistent and emphatic group of protective antigens to date identified from blood-feeding parasites are those expressed on the surface of the gut and presumed to function in blood meal digestion. Gut membrane proteins have been effectively employed as vaccine antigens against cattle ticks [23] and $\mathrm{H}$. contortus [38, 44] and are offering promise as vaccine components against hookworms. Haemoglobin digestion is thought to be facilitated by a multi-enzyme cascade in blood-feeding parasites [45, 69] and some of the enzymes involved in this process have been identified in hookworms and Haemonchus with many commonalities evident between the two and also when compared to the fluke and malaria parasites. Vaccination of dogs with the cysteine haemoglobinase, Ac-CP-2, showed that haemoglobinolytic proteases expressed in recombinant form are efficacious vaccines against hookworm infections [70] with adult hookworms recovered from the intestines of dogs vaccinated with CP-2 being stunted and the number of female hookworms significantly reduced compared to control dogs. Ac-APR-1, an aspartic haemoglobinase from A. caninum, and its orthologue from N. americanus, NaAPR-1, were expressed in catalytically active form, and cleaved host haemoglobin at the hinge region among many other sites [71]. Vaccination of dogs with recombinant AcAPR-1 significantly reduced hookworm burdens and faecal egg counts and these dogs were protected against blood loss and did not develop anaemia [72]. Like the Ac-CP-2 vaccine, IgG from animals vaccinated with APR-1 inhibited enzyme activity in vitro, and antibody bound in situ to the intestines of worms recovered from vaccinated dogs, implying that the vaccine interferes with the parasite's ability to digest blood. This was the first report of a recombinant vaccine from a haematophagous parasite that significantly reduced both parasite load and blood loss, supporting the development of APR-1 as a second arm of the HHV. A catalytically inactive form of a homologue of APR-1 found in Necator americanus also induced protection against $\mathrm{A}$. caninum in dogs. Residents in high-transmission areas for N. americanus had high circulating IgG1 to the inactive Na-APR mutant, indicating that natural boosting may occur in exposed humans [73].

\section{MALARIA}

Malaria is the world's most important tropical parasitic disease. The worldwide prevalence of the disease is in the order of 350-500 million clinical cases each year, with an estimated annual death toll of over 1.1 million deaths (WHO 2008 Immunization, Vaccines and Biologicals). Significant economic costs arise from treatment and days of labour lost. The parasite has a complex lifecycle initiated when female mosquitoes introduce sporozoites into the bloodstream when taking a blood meal which then migrate to the liver and invade hepatocytes. Here they develop into merozoites with tens of thousands being produced over a period of about 2 weeks. The merozoites emerge from the hepatocytes and enter the bloodstream where they invade erythrocytes (RBCs), multiply and mature within 24-72 hours after which the RBCs lyse releasing the merozoites which immediately infect new RBCs to repeat the cycle. The classical clinical signs of malaria, acute febrile episodes and rigors that occur every 48 to 72 hours, coincide with the synchronized lysis of infected RBCs releasing the newly matured merozoites (WHO). Some merozoites develop into sexual-stage gametocytes, which can sexually combine and develop into new sporozoites when taken up by an anopheline mosquito, thus reinitiating the cycle.

Immunization of human subjects with irradiated sporozoites confers high levels $(\sim 90 \%)$ of protection against experimental infection (e.g. [74]) suggesting that vaccination is feasible. Individuals in malaria-endemic countries do acquire natural immunity that builds up slowly requires continual antigenic stimulation and affects the severity of disease (WHO 2008). Protection has been elicited by passive transfer of hyperimmune immunoglobulins from malariaimmune adults into malaria-naïve human volunteers [75]. There are three basic approaches to malaria vaccination 1) to stimulate strong immune responses that can prevent merozoites entering the blood (pre-erythrocytic) 2) vaccination which induces disease-limiting immunity and 3) population-based immunization aimed at preventing subsequent mosquito transmission [76].

Pre-erythrocytic vaccine strategies aim to generate an antibody response that will neutralize sporozoites and prevent them from invading the hepatocyte, and/or to elicit a cell-mediated immune response that will inhibit intra-hepatic parasites. This type of vaccine would prevent the advent of clinical disease. The most advanced pre-erythrocytic (liverstage) vaccine candidate is derived from the circumsporozoite protein (CSP), the major component of the surface of the sporozoite Prototype vaccines were designed to induce antibody responses against the repeat epitopes of the circumsporozoite protein and provided the first evidence that humans could be protected from malaria infection with a subunit vaccine $[77,78]$. The candidate vaccines were poorly immunogenic and a vaccine was developed comprising a hybrid of the circumsporozoite protein fused to hepatitis B surface antigen (HBsAg) expressed together with unfused HBsAg [79]. From these prototypes, RTS,S/AS02 arose which is composed of both the CSP repeat antibody targets as well as C-terminal non-repeat regions that are targets for cell-mediated immunity [80]. These were fused to the hepatitis B surface antigen and expressed in yeast [81]. Initial Phase I clinical trials of RTS,S formulated with Glaxo Smith-Kline AS02 adjuvant, an adjuvant vehicle which consists of a lipid emulsion, showed protection against malaria challenge in six out of seven volunteers (WHO 2008). More recently, the vaccine has been formulated with AS01, a liposomal vehicle, and this vaccine induced higher levels of antibody and Th1 responses to the CS protein [81]. Vaccination with these formulations protects against infection and reduces blood parasitemia. Extensive clinical studies are under way, including one aimed at combining RTS,S with a blood-stage antigen MSP-1 (summarized in 
some detail in [80]. MSP-1 is expressed on the merozoite surface and is also a target for protective immunity [82].

Another leading candidate is an apical membrane antigen 1 (AMA-1), a type I transmembrane protein located in the micronemes of the merozoite that is functional in the rapid invasion of erythrocytes [83]. Preclinical studies showed that vaccination with AMA-1 induces antibodies and protection against homologous parasite challenge in both rodent and monkey models of malaria infection [84, 85]. Vaccination of monkeys with recombinant Plasmodium falciparum apical membrane antigen 1 confers protection against blood-stage malaria. The outcome of a phase $1 / 2$ a study which evaluated the safety, immunogenicity, and efficacy of a vaccine composed of the recombinant Plasmodium falciparum AMA-1 representing the 3D7 allele formulated with either the AS01B or AS02A Adjuvant Systems has just been reported [86]. All vaccine formulations stimulated similar functional antibody responses, as judged using a growth inhibition assay against homologous parasites and demonstrable interferon-gamma (IFN-gamma) responses. However, volunteers challenged with P. falciparum-infected mosquitoes all became parasitemic although a small but significant reduction of parasitemia in the AMA-1/AS02A group was noted.

Asexual blood-stage (erythrocytic) vaccine strategies aim to elicit antibodies that will inactivate merozoites and/or target malarial antigens expressed on the RBC surface, thus inducing antibody-dependent cellular cytotoxicity and complement lysis; they also are meant to elicit T-cell responses that will inhibit the development of the parasite in RBCs. This type of vaccine would mostly serve as a diseasereduction vaccine in endemic countries by decreasing the exponential multiplication of merozoites. The development of MSP-1 as a vaccine candidate is the subject of a recent detailed review [82]. Briefly, MSP1 conferred protection against challenge infection against Plasmodium yoelii in laboratory mice [87] and passive immunization with monoclonal antibodies (mAbs) also provided protection in the same model, highlighting the importance of antibody for protection [88, 89]. Gene sequence analysis [90] allowed detailed structural analysis which identified a disulphide-rich region of approximately 100 amino acids at the C-terminus which encoded 2 epidermal growth factor (EGF) domains [82]. Subsequent work showed that immunization with recombinant forms of the EGF domains protected against challenge infection with blood-stage rodent parasites [91]. Evidence that MSP-1 is important in natural acquired immunity and can consistently confer protective immunity is equivocal [82].

Transmission-blocking vaccines induce antibodies against the sexual stage antigens which prevent the development of infectious sporozoites in the salivary glands of the Anopheles mosquitoes. The leading candidate vaccines contain the $P$. falciparum surface protein antigens Pfs 25 and Pfs 28 or their P. vivax homologues Pvs 25 and Pvs28 [92]. These vaccines are currently being developed at the NIH as recombinant yeast-secreted proteins (S. cerevisiae). Initial human Phase I trials have been conducted for Pfs25 and should follow soon for Pvs25. Other sexual stage-specific antigens that are being developed as transmission-blocking vaccines are Pfs $48 / 45$ and Pfs 230 .
The concept of attenuation and parasite challenge to elicit immunity is also being explored. As noted earlier, an attempt to develop a commercial attenuated sporozoite vaccine has been undertaken by Sanaria Inc., with the support from the Bill and Melinda Gates Foundation and the NIH and the outcome of clinical trials is awaited.

\section{LEISHMANIA}

Leishmaniasis, caused by several species of flagellated protozoan parasites of the Leishmania genus, is prevalent in Africa, Latin America, South and Central Asia, the Mediterranean basin and the Middle East. There are an estimated 12 million cases annually (WHO) and disease can cause serious disfigurement as well as death. Parasites are usually transmitted from a wild-animal reservoir (small rodents, dogs) by biting sand flies although transmission can occur from infected humans. The disease has cutaneous, mucocutaneous and visceral forms. About $1.5-2$ million occur annually and epidemics of visceral leishmaniasis can arise with high mortality rates. Treatment relies on chemotherapy, is expensive and becoming compromised by emerging drug resistance. Recovery from infection renders an individual resistant to subsequent infection indicating that a successful vaccine is feasible [93]. As noted earlier, vaccination is possible with by controlled infection using infectious material from lesions [17] but the use of live virulent organisms for vaccination was largely discontinued and in the 1990s the focus shifted to attenuated organisms. Attenuated parasite vaccines are likely to mimic natural infection and hence induce an appropriate immune response. However, there are issues of transient infection only delivering small amounts of antigen leading to the possibility of inappropriate skew in the bias of the Th1 response. A possible advantage is that such immunization will also deliver many more parasite antigens than the limited number possible with subunit or recombinant antigens [17].

Various subunit recombinant candidate vaccines have been tested in murine models of cutaneous Leishmaniasis and this work was extensively reviewed in [93] and summarized below. A lead candidate is a surface-expressed glycoprotein gp63, or leishmanolysin. This is a zinc metalloprotease [94] which is expressed on the promastigote surface and mediates internalisation of promastigotes. Efficacy with recombinant versions has been patchy [90]. Gp63 expressed in E. coli induced partial protection in monkeys [95]. However, the native protein purified form L. major did protect mice against challenge with either $\mathrm{L}$. mexicana or L. major and protection was evident when gp63 was expressed in BCG [96] and attenuated Salmonella [97]. Studies in mice indicated that host genetics influence the inititiation of protective immune responses to the vaccine [93]. DNA vaccination is considered an attractive option for vaccine development since this approach generally leads to the induction of Th1 responses [98]. A variety of DNA vaccine constructs have been tested with variable outcomes. In addition, some protective immunity can be stimulated by vaccination with sand fly salivary proteins, sand flies being the insect vector for the parasite with a $15 \mathrm{kDa}$ protein showing promise [99].

Whilst considerable progress has been made it is informative to quote [93] Kedzierski et al. 2006 regarding 
the definition of an ideal antileishmanial vaccine. "One of the requirements of an "ideal" antileishmanial vaccine is to be effective against more than one Leishmania species in order to protect individuals in areas where cutaneous and visceral leishmaniasis, for example, coexist". Recent evidence (e.g. [100]) suggests that cross-protection is possible. It is of interest to note that Summarizing a large amount of experimental evidence, Rivier et al. [101] concluded that injection of attenuated organisms achieved better protection than any method involving recombinant gp63 as test antigen delivered with a variety of adjuvants and delivery systems. If this conclusion is generally applicable to other vaccine candidates, the prospect of using attenuated Leishmania vaccines in preference to subunit or recombinant approaches is likely to come to the fore although there would be problems of large-scale production and distribution in the field.

\section{STAGE-SPECIFIC ANTIGEN EXPRESSION AND ANTIGENIC VARIATION}

Protozoan and metazoan parasites present a variety of temporally expressed antigens to the host immune system which also show stage-specificity. Some will be associated with protective immune responses, some not. The major challenge for the vaccinologist is to identify those required for the expression of protective immunity. Moreover, all parasites show considerable antigenic variation and this is exemplified by the variant surface glycoprotein (VSG) of trypanosomes, the causative agent of sleeping sickness which is transmitted by tetse flies and is particularly prevalent in Africa. VSGs are densely packed on the parasite surface and physically protect underlying proteins from effectors of the host immune system [102]. This surface coat elicits a specific, trypanocidal immune response and this is countered by antigenic variation, in which trypanosomes switch to expression of a distinct VSG which, if antigenically novel, allows clonal proliferation of the switched cells, generating a new parasitaemia peak. Each trypanosome expresses only one VSG gene but has the potential to switch to any of probably hundreds of others [103].

\section{ANIMAL MODELS AND VACCINE DEVELOPMENT}

In many parasitic diseases, parasite vaccine development is highly dependent on the use of animal models, firstly to define the immune responses which may mediate protection and, secondly, to evaluate the protective efficacy (and safety) of candidate antigens prior to clinical trials. There are many caveats to this approach. For example, candidate antigens which show efficacy in an animal model are often less effective or even ineffective in the natural host. The animal model may not be fully permissive with the result that infection outcomes are subtly different from those in the natural host. Rodent models are in routine use yet the data can be contradictory and potentially misleading. Studies of $S$. mansoni infection in rats and humans indicated that a vaccine which exploits Th2-based effector mechanisms may be most appropriate while vaccination studies in mice indicated that T-cell-mediated responses involving IFN- $\gamma$ and IL-12-dependent mechanisms might be required [104](Wilson \& Coulson, 1999). Work in double cytokinedeficient mice indicated that highly polarized Th1- and Th2- type cytokine and antibody responses contribute equally to vaccine-induced immunity to $S$. mansoni [105] (Hoffmann et al. 1999). However, despite these caveats, animal models have proven invaluable in vaccine development against parasitic disease.

\section{CONTINUED ANTIGEN DISCOVERY -EXPLOITING THE GENOME REVOLUTION}

Parasite genome sequencing projects are rapidly providing the resource to define the full potential protein complement of a parasite and, using transcriptome analysis platforms such as microarray, to correlate parasite gene expression with life cycle requirements and to define how a parasite might adapt to evade the host immune response. Downstream bioinformatic analyses followed by in vitro and in vivo hypothesis testing should prove invaluable for identifying further potential vaccine antigens. For example, databases can be mined with set criteria such as i) secreted proteins, ii) conservation between species, but not in the host genome and iii) abundancy in the parasite stage of interest. This approach was applied to the malaria sequence database and identified a number of novel and potential vaccine candidates which merit further investigation [106]. Another approach is to identify proteins secreted by the parasite or expressed on a surface which may be accessible to the host immune system [107]. Model organisms are being used to identify potential key genes required for parasite survival. The best example of this is the application of the C. elegans genome to identify targets in parasitic counterparts. The function of most $C$. elegans genes has been defined using traditional reverse genetics approaches and RNA interference (RNAi). Many of the genes have close homologues in parasitic nematodes and the hope is to identify gene knockdown which results in a lethal phenotype would point at parasite homologues which may be useful vaccine targets [14]. The development of RNAi in parasites (e.g. [108-112]) provides the possibility of defining if a vaccine target is critical for parasite survival by specific gene-knockdown. It is really beyond the scope of this review to explore the potential and pitfalls associated with genome analysis and downstream exploitation but a general search in Pubmed (www.ncbi.nlm.nih.gov/sites/entrez) with the search string words genomes, vaccine discovery, parasite, review, will lead the reader to a number of informative review articles.

\section{SINGLE OR MULTI-VALENT VACCINES?}

The efficacy achieved by vaccination often falls short of that required to control the disease. Knox and Redmond [14] posed the question "should we focus more on testing antigen cocktails?" and commented that parasites are complex organisms and it is probably naïve to think that a protective immune response could be stimulated by vaccination with a single protein. A cautionary example is that cattle vaccinated with a combination of two recombinant oncosphere proteins of $T$. saginata were almost completely protected against experimental challenge infection whereas, neither antigen was protective when tested individually [113]. Other examples that antigen combinations may be more effective are a series of vaccination trials against $F$. hepatica in cattle which showed that a combination of cathepsin L1 and haemoglobin gave significantly higher levels of protection 
against challenge compared to animals immunised with either antigen alone [114]. Similar enhanced combination effects have been described in vaccination studies against cattle ticks (B. microplus; [115]) and hookworm [116]. DNA vaccination provides a method to deliver antigen combinations by fusing genes encoding individual antigens together in a single construct. This approach is being evaluated for Leishmania [117] and malaria [118], the constructs targeting more than one parasite stage. DNA vaccine constructs can also include immunomodulators such as $\mathrm{CpG}$ motifs and cytokines which may drive an appropriate immune response [119].

\section{RECOMBINANT PROTEIN PRODUCTION}

A wide variety of pro- and eukaryotic vectors are widely available for expression of recombinant proteins but which one do you choose at the outset of a vaccination study? This choice will be influenced by knowledge of the antigen in question. Is correct protein folding a pre-requisite for efficacy and/or does post-translational modification contribute to driving a protective response? Bacteria do not glycosylate proteins while the nature of yeast and insect cell glycan may be inappropriate [14]. These authors noted that experiments had shown that the bulk of the circulating antibody response of sheep antibody response to protective gut antigens from Haemonchus contortus was to the glycan component but that current evidence suggested that this was unlikely to be contributing to protection associated with the vaccine [120]. Parasite-specific patterns of glycosylation have been identified, e.g. H. contortus H11 [121], and the currently-available commercial eukaryotic expression systems will not result in appropriate glycosylation of recombinant antigens. Recent work has explored the possibility of expressing parasitic nematode-derived genes in the free-living nematode Caenorhabditis elegans (see [122, 123]) to overcome these difficulties.

\section{ADJUVANTS AND ANTIGEN DELIVERY}

It would not be possible, within the limitations of the present discussion, to address adequately the issues of adjuvants and antigen delivery. The interested reader should refer to a vast variety of reviews on these topics available in public databases on the world-wide web. Suffice to say here that the wrong choice of adjuvant can lead to misleading experimental outcomes with the result that good vaccine candidates may be rejected simply because inappropriate adjuvanting or delivery was used in the initial phases of testing. This is exemplified by a body of work investigating adult ES products as vaccine candidates against $O$. ostertagi in cattle [124]. One component, an ASP homologue was efficacious when given intramuscularly with QuilA as adjuvant but had no effect with aluminium hydroxide as adjuvant [124].

\section{APPLYING VACCINES}

Even when a vaccine with the required efficacy comes through exhaustive laboratory and field trials, a number of hurdles before final uptake and use remain. The reader is directed to accounts of this process in the livestock sector with vaccines against Boophilus microplus [23] and vaccines against cestodes [24]. Hotez et al. [125] listed a number of obstacles which would need to be addressed before a hookworm vaccine could become a reality. These included hookworm-induced immunosuppression, the effects of coinfection with other helminths, cross-reactivity to autoantigens and toxicity of the protective response. Then there is the issue of who pays to deliver the vaccine to the target population. Parasitic diseases are most prevalent in tropical and sub-tropical regions of the world and, hence, impact severely on the "poorest of the poor". Economic impacts are compounded by a reluctance of travelers to visit endemic areas. For example, the control and eradication of malaria remains a priority and without doubt an effective vaccine would represent be cost-effective [126]. There is unlikely to be a commercial market for a vaccine in these regions of most need. Vaccine production may be carried out on a regional basis and funded by local governments, international aid agencies or by philanthropists [14]. The vast increase in global travel, be it tourism, business or military of course may alter the economic equation relating to vaccine profitability with malaria being a clear example. For livestock and companion animals, vaccine development is likely to be driven by the commercial pressures of the market place. The issues around vaccine development to counter parasite disease of man and animals have been discussed recently in a special supplement of the journal Parasitology [127]. As noted earlier, the introduction of a parasite vaccine is likely to require a considerable effort in education. A vaccine is unlikely to induce sterile immunity but, more likely, to minimize the impact of infection which, in the agricultural sector can probably be defined a eliminating or minimizing the economic impact of the disease. In some areas where drug resistance is rife, this may also translate into the only option to sustain livestock production.

\section{ACKNOWLEDGEMENTS}

The author gratefully acknowledges funding from the Scottish Executive Environment and Rural Affairs Department and the global research community.

\section{REFERENCES}

[1] Kaplan R. Drug resistance in nematodes of veterinary importance. a status report. Trends Parasitol 2004; 20: 477-81.

[2] Fallon PG, Sturrock RF, Niang AC, Doenhoff MJ. Short report. diminished susceptibility to praziquantel in a Senegal isolate of Schistosoma mansoni. Am J Trop Med Hyg 1995; 53: 61-2.

[3] Ismail M, Metwally A, Farghaly A, Bruce J, Tao LF, Bennett JL. Characterization of isolates of Schistosoma mansoni from Egyptian villagers that tolerate high doses of praziquantel. Am J Trop Med Hyg 1996; 55: 214-8.

[4] De Clercq D, Sacko M, Behnke J, Gilbert F, Dorny P, Vercruysse J. Failure of mebendazole in treatment of human hookworm infections in the southern region of Mali. Am J Trop Med Hyg 1997; 57: 25-30.

[5] Coppel RL, Brown GV, Mitchell GF, Anders RF, Kemp DJ. Identification of a cDNA clone encoding a mature blood stage antigen of Plasmodium falciparum by immunization of mice with bacterial lysates. EMBO J 1984; 3: 403-7.

[6] Enea V, Ellis J, Zavala F, et al. DNA cloning of Plasmodium falciparum circumsporozoite gene. amino acid sequence of repetitive epitope. Science 1984; 225: 628-30.

[7] Sibley $\mathrm{CH}$, Hunt SY. Drug resistance in parasites. can we stay ahead of the evolutionary curve? Trends Parasitol 2003; 19: 532-7.

[8] van der Werf MJ, de Vlas SJ, Brooker S, et al. Quantification of clinical morbidity associated with schistosome infection in subSaharan Africa. Acta Trop 2003; 86: 125-39. 
[9] McManus DP, Li Y, Gray DJ, Ross AG. Conquering 'snail fever'. schistosomiasis and its control in China. Expert Rev Anti-Infect Ther 2009; 7: 473-85.

[10] McManus DP, Dalton JP. Vaccines against the zoonotic trematodes Schistosoma japonicum, Fasciola hepatica and Fasciola gigantica. Parasitology 2006; 133 Suppl: S43-61.

[11] Mas-Coma S, Bargues MD, Valero MA. Fascioliasis and other plant-borne trematode zoonoses. Int J Parasitol 2005; 35: 1255-78.

[12] Esteban JG, Gonzalez C, Curtale F, et al. Hyperendemic fascioliasis associated with schistosomiasis in villages in the Nile Delta of Egypt. Am J Trop Med Hyg 2003; 69: 429-37.

[13] Marcos LA, Terashima A, Gotuzzo E. Update on hepatobiliary flukes. fascioliasis, opisthorchiasis and clonorchiasis. Curr Opin Infect Dis 2008; 21: 523-30.

[14] Knox DP, Redmond DL. Parasite vaccines - recent progress and problems associated with their development. Parasitology 2006; 133 (Suppl): S1-8.

[15] Vercruysse J, Knox DP, Schetters TP, Willadsen P. Veterinary parasitic vaccines. pitfalls and future directions. Trends Parasitol 2004; 20: 488-92.

[16] Uilenberg G. Immunization against diseases caused by Theileria parva. a review. Trop Med Int Health 1999; 4: A12-20.

[17] Handman E. Leishmaniasis. current status of vaccine development. Clin Microbiol Rev 2001; 14: 229-43.

[18] Hoffman SL, Goh LM, Luke TC, et al. Protection of humans against malaria by immunization with radiation-attenuated Plasmodium falciparum sporozoites. J Infect Dis 2002; 185: 115564.

[19] Jarrett WF, Jennings FW, McIntyre WI, Mulligan W, Urquhart GM. Immunological studies on Dictyocaulus viviparus infection; active immunization with whole worm vaccine. Immunology 1960 3: $135-44$

[20] Barnes EH, Dobson RJ, Barger IA. Worm control and anthelmintic resistance. adventures with a model. Parasitol Today 1995; 11: 5663.

[21] Williams GM, Sleigh AC, Li Y, et al. Mathematical modelling of schistosomiasis japonica. comparison of control strategies in the People's Republic of China. Acta Trop 2002; 82: 253-62.

[22] Wynn TA, Hoffman KF. Defining a schistosomiasis vaccination strategy - is it really Th1 versus Th2? Parasitol Today 2000; 16: 497-501.

[23] Willadsen P. Vaccination against ectoparasites. Parasitology 2006; 133: S9-S25.

[24] Lightowlers MW. Cestode vaccines. origins, current status and future prospects. Parasitology 2006; 133: S27-42.

[25] Knox DP. Development of vaccines against gastrointestinal nematodes. Parasitology 2000; 120: S43-S61.

[26] Johnson KS, Harrison GB, Lightowlers MW, et al. Vaccination against ovine cysticercosis using a defined recombinant antigen. Nature 1989; 338: 585-7.

[27] Harrison GB, Heath DD, Dempster RP, et al. Identification and cDNA cloning of two novel low molecular weight host- protective antigens from Taenia ovis oncospheres. Int J Parasitol 1996; 26: 195-204.

[28] Lightowlers MW, Rolfe R, Gauci CG. Taenia saginata. vaccination against cysticercosis in cattle with recombinant oncosphere antigens. Exp Parasitol 1996; 84: 330-8.

[29] Flisser A, Gauci CG, Zoli A, et al. Induction of protection against porcine cysticercosis by vaccination with recombinant oncosphere antigens. Infect Immun 2004; 72: 5292-7.

[30] Gonzalez AE, Gauci CG, Barber D, et al. Vaccination of pigs to control human neurocysticercosis. Am J Trop Med Hyg 2005; 72 : 837-9.

[31] Heath DD, Lawrence SB. 1996 Antigenic polypeptides of Echinococcus granulosus oncospheres and definition of protective molecules. Para Immunol 1996; 18: 347-57.

[32] Willadsen P, Riding GA, McKenna R V, et al. Immunologic control of a parasitic arthropod. Identification of a protective antigen from B. microplus. J Immunol 1989; 143: 1346-51.

[33] Rand KN, Moore T, Sriskantha A, et al. Cloning and expression of a protective antigen from the cattle tick $B$. microplus. Proc Natl Acad Sci USA 1989; 86: 9657-61.

[34] Gough JM, Kemp DH. Localization of a low abundance membrane protein (Bm86) on the gut cells of the cattle tick B. microplus by immunological labelling. J Parasitol 1993; 79: 900-907.

[35] Willadsen P. Anti-tick vaccines. Parasitology 2004; 129: S367-87.
[36] Knox DP, Redmond DL, Newlands GF, Skuce PJ, Pettit D, Smith WD. The nature and prospects for gut membrane proteins as vaccine candidates for Haemonchus contortus and other ruminant trichostrongyloids. Int J Parasitol 2003; 33: 1129-37.

[37] Bethony JM, Loukas A, Hotez PJ, Knox DP. Vaccines against blood-feeding nematodes of humans and livestock. Parasitology 2006;133 Suppl: S63-79.

[38] Knox DP, Smith WD. Vaccination against gastrointestinal nematode parasites of ruminants using gut-expressed antigens. Vet Parasitol 2001; 100: 21-32.

[39] Newton SE, Munn EA. The development of vaccines against gastrointestinal nematode parasites, particularly Haemonchus contortus. Parasitol Today 1999; 15: 116-22.

[40] Smith WD, Smith S. Murray JM. Protection studies with integral membrane fractions of Haemonchus contortus. Para Immunol 1994; 16: 231-41.

[41] Longbottom D, Redmond DL, Russell M, Liddell S, Smith WD, Knox DP. Molecular cloning and characterisation of a putative aspartate proteinase associated with a gut membrane protein complex from adult Haemonchus contortus. Mol Biochem Parasitol 1997; 88: 63-72.

[42] Redmond DL, Knox DP, Newlands G, Smith WD. Molecular cloning and characterisation of a developmentally regulated putative metallopeptidase present in a host protective extract of Haemonchus contortus. Mol Biochem Parasitol 1997; 85: 77-87.

[43] Loukas A, Bethony JM, Williamson AL, et al. Vaccination of dogs with a recombinant cysteine protease from the intestine of canine hookworms diminishes the fecundity and growth of worms. J Infect Dis 2004; 189: 1952-61.

[44] Loukas A, Bethony JM, Mendez S, et al. Vaccination with recombinant aspartic hemoglobinase reduces parasite load and blood loss after hookworm infection in dogs. PLoS Med 2005; 2: e295.

[45] Goldberg DE. Haemoglobin degradation. Curr Top Microbiol Immunol 2005; 295: 275-291.

[46] Williamson AL, Brindley PJ, Knox DP, Hotez PJ, Loukas A. Digestive proteases of blood-feeding nematodes. Trends Parasitol 2003; 19: 417-23.

[47] Williamson AL, Lecchi P, Turk BE, et al. A multi-enzyme cascade of hemoglobin proteolysis in the intestine of blood-feeding hookworms. J Biol Chem 2004; 279: 3560-67.

[48] McManus DP, Loukas A. Current status of vaccines for schistosomiasis. Clin Microbiol Rev 2008; 21: 225-42.

[49] Tran MH, Pearson MS, Bethony JM, et al. Tetraspanins on the surface of Schistosoma mansoni are protective antigens against schistosomiasis. Nat Med 2006; 12: 835-40.

[50] Da'dara AA, Skelly PJ, Wang MM, Harn DA. Immunization with plasmid DNA encoding the integral membrane protein, Sm23, elicits a protective immune response against schistosome infection in mice. Vaccine 2001 12; 20: 359-69.

[51] Pearce EJ, James SL, Hieny S, Lanar DE, Sher A. Induction of protective immunity against Schistosoma mansoni by vaccination with Schistosome paramyosin (Sm97), a nonsurface parasite antigen. Proc Natl Acad Sci USA 1988 ; 85: 5678-82.

[52] Harn DA, Gu W, Oligino LD, Mitsuyama M, Gebremichael A, Richter D. A protective monoclonal antibody specifically recognizes and alters the catalytic activity of schistosome triosephosphate isomerase. J Immunol 1992; 148: 562-7.

[53] Zhu Y, Si J, Harn DA, et al. Schistosoma japonicum triosephosphate isomerase plasmid DNA vaccine protects pigs against challenge infection. Parasitology 2006; 132: 67-71.

[54] $\mathrm{Yu} \mathrm{XL}, \mathrm{He} \mathrm{YK}, \mathrm{Xiong} \mathrm{T}$, et al. Protective effects of coimmunization with SjCTPI-Hsp70 and interleukin-12 DNA vaccines against Schistosoma japonicum challenge infection in water buffalo. Zhongguo Ji Sheng Chong Xue Yu Ji Sheng Chong Bing Za Zhi 2006; 24: 433-6.

[55] Balloul JM, Grzych JM, Pierce RJ, Capron A. A purified 28,000 dalton protein from Schistosoma mansoni adult worms protects rats and mice against experimental schistosomiasis. J Immunol 1987;138: 3448-53.

[56] Boulanger D, Reid GD, Sturrock RF, et al. Immunization of mice and baboons with the recombinant Sm28GST affects both worm viability and fecundity after experimental infection with Schistosoma mansoni. Parasite Immunol 1991;13: 473-90. 
[57] Capron A, Riveau G, Capron M, Trottein F. Schistosomes. The road from host-parasite interactions to vaccines in clinical trials. Trends Parasitol 2005; 21: 143-9.

[58] Tendler M, Vilar MM, Brito CA, Freire NM, Katz N, Simpson A. Vaccination against schistosomiasis and fascioliasis with the new recombinant antigen Sm14. potential basis of a multi-valent antihelminth vaccine? Mem Inst Oswaldo Cruz 1995; 90: 255-6.

[59] Brito CF, Oliveira GC, Oliveira SC, et al. Sm14 gene expression in different stages of the Schistosoma mansoni life cycle and immunolocalization of the Sm14 protein within the adult worm. Braz J Med Biol Res 2002; 35: 377-81.

[60] Brindley PJ, Pearce EJ. Genetic manipulation of schistosomes. Int J Parasitol 2007; 37: 465-73.

[61] de Silva NR, Brooker S, Hotez PJ, Montresor A, Engels D, Savioli L. Soil-transmitted helminth infections. updating the global picture. Trends Parasitol 2003; 19: 547-51.

[62] Bethony J, Brooker S, Albonico M, et al. Soil-transmitted helminth infections. ascariasis, trichuriasis, and hookworm. Lancet 2006; 367: 1521-32.

[63] Evans AC, Stephenson LS. Not by drugs alone. the fight against parasitic helminths. World Health Forum 1995; 16(3): 258-61.

[64] Bethony JM, Loukas A, Hotez PJ, Knox DP. Vaccines against blood-feeding nematodes of humans and livestock. Parasitology 2006; 133 (Suppl): S63-79.

[65] Bethony J, Loukas A, Smout M, et al. Antibodies against a secreted protein from hookworm larvae reduce the intensity of hookworm infection in humans and vaccinated laboratory animals. FASEB J 2005; 19: 1743-5

[66] Sen L, Ghosh K, Bin Z, et al. Hookworm burden reductions in $\mathrm{BALB} / \mathrm{c}$ mice vaccinated with recombinant Ancylostoma secreted proteins (ASPs) from Ancylostoma duodenale, Ancylostoma caninum and Necator americanus. Vaccine 2000; 18: 1096-102.

[67] Goud GN, Zhan B, Ghosh K, et al. Cloning, yeast expression, isolation, and vaccine testing of recombinant Ancylostoma-secreted protein (ASP)-1 and ASP-2 from Ancylostoma ceylanicum. J Infect Dis 2004; 189: 919-29.

[68] Bethony JM, Simon G, Diemert DJ, et al. Randomized, placebocontrolled, double-blind trial of the Na-ASP-2 hookworm vaccine in unexposed adults. Vaccine 2008; 26: 2408-17.

[69] Williamson AL, Brindley PJ, Knox DP, Hotez PJ, Loukas A. Digestive proteases of blood-feeding nematodes. Trends Parasitol 2003; 19: 417-23.

[70] Loukas A, Bethony JM, Williamson AL, et al. Vaccination of dogs with a recombinant cysteine protease from the intestine of canine hookworms diminishes the fecundity and growth of worms. J Infect Dis 2004;189: 1952-61.

[71] Williamson AL, Lecchi P, Turk BE, et al. A multi-enzyme cascade of hemoglobin proteolysis in the intestine of blood-feeding hookworms. J Biol Chem 2004; 279: 35950-7.

[72] Loukas A, Bethony JM, Mendez S, et al. Vaccination with recombinant aspartic hemoglobinase reduces parasite load and blood loss after hookworm infection in dogs. PLoS Med 2005; 2 : e295.

[73] Pearson MS, Bethony JM, Pickering DA, et al. An enzymatically inactivated hemoglobinase from Necator americanus induces neutralizing antibodies against multiple hookworm species and protects dogs against heterologous hookworm infection. FASEB J 2009; 23: 3007-19.

[74] Luke TC, Hoffman SL. Rationale and plans for developing a nonreplicating, metabolically active, radiation-attenuated Plasmodium falciparum sporozoite vaccine. J Exp Biol 2003; 206: 3803-8.

[75] McGregor IA. The passive transfer of human malarial immunity. Am J Trop Med Hyg 1964; 13: Suppl 237-9.

[76] Ann Stewart V, Coppel R. Issues in malaria vaccine development. Parasite Immunol 2009;31: 489-91.

[77] Hoffman SL, Wistar R Jr, Ballou WR, et al. Immunity to malaria and naturally acquired antibodies to the circumsporozoite protein of Plasmodium falciparum. N Engl J Med 1986 315: 601-6

[78] Ballou WR, Hoffman SL, Sherwood JA, et al. Safety and efficacy of a recombinant DNA Plasmodium falciparum sporozoite vaccine. Lancet 1987; 1(8545): 1277-81

[79] Stoute JA, Slaoui M, Heppner DG, et al. A preliminary evaluation of a recombinant circumsporozoite protein vaccine against Plasmodium falciparum malaria. RTS,S Malaria Vaccine Evaluation Group. N Engl J Med 1997; 336: 86-91.
[80] Ballou WR. The development of the RTS,S malaria vaccine candidate. challenges and lessons. Parasite Immunol 2009; 31: 492500.

[81] Garçon N, Heppner DG, Cohen J. Development of RTS,S/AS02. a purified subunit-based malaria vaccine candidate formulated with a novel adjuvant. Expert Rev Vaccines 2003; 2: 231-8.

[82] Holder AA. The carboxy-terminus of merozoite surface protein 1. Structure, specific antibodies and immunity to malaria. Parasitology 2009; 136: 1445-56.

[83] Treeck M, Zacherl S, Herrmann S, et al. Functional analysis of the leading malaria vaccine candidate AMA-1 reveals an essential role for the cytoplasmic domain in the invasion process PLoS Pathog. 2009 5(3): e1000322.

[84] Collins WE, Pye D, Crewther PE, et al. Protective immunity induced in squirrel monkeys with recombinant apical membrane antigen-1 of Plasmodium fragile. Am J Trop Med Hyg 1994 51: 711-9.

[85] Stowers AW, Kennedy MC, Keegan BP, Saul A, Long CA, Miller LH. Vaccination of monkeys with recombinant Plasmodium falciparum apical membrane antigen 1 confers protection against blood-stage malaria. Infect Immun 2002 70: 6961-7.

[86] Spring MD, Cummings JF, Ockenhouse CF, et al. Phase $1 / 2$ a study of the malaria vaccine candidate apical membrane antigen-1 (AMA-1) administered in adjuvant system AS01B or AS02A. PLoS One 2009; 4(4): e5254.

[87] Freeman RR, Holder AA. Characteristics of the protective response of BALB/c mice immunized with a purified Plasmodium yoelii schizont antigen. Clin Exp Immunol 1983; 54: 609-16.

[88] Majarian WR, Daly TM, Weidanz WP, Long CA. Passive immunization against murine malaria with an IgG3 monoclonal antibody. J Immunol 1984; 132: 3131-7.

[89] Spencer Valero LM, Ogun SA, Fleck SL, et al. Passive immunization with antibodies against three distinct epitopes on Plasmodium yoelii merozoite surface protein 1 suppresses parasitemia. Infect Immun 1998; 66: 3925-30.

[90] Holder AA, Blackman MJ, Burghaus PA, Chappel JA, Ling IT, McCallum-Deighton N, Shai S A malaria merozoite surface protein (MSP1)-structure, processing and function. Mem Inst Oswaldo Cruz 1992; 87 (Suppl) 3: 37-42.

[91] Daly TM, Long CA. Humoral response to a carboxyl-terminal region of the merozoite surface protein-1 plays a predominant role in controlling blood-stage infection in rodent malaria. J Immunol $1995 ; 155: 236-43$

[92] $\mathrm{Wu} \mathrm{Y,} \mathrm{Ellis} \mathrm{RD,} \mathrm{Shaffer} \mathrm{D,} \mathrm{et} \mathrm{al.} \mathrm{Phase} 1$ trial of malaria transmission blocking vaccine candidates Pfs25 and Pvs25 formulated with montanide ISA 51. PLoS One 2008; 3(7): e2636.

[93] Kedzierski L, Zhu Y, Handman E. Leishmania vaccines: progress and problems. Parasitology 2006; 133 Suppl: S87-112.

[94] Chaudhuri G, Chaudhuri M, Pan A, Chang KP. Surface acid proteinase (gp63) of Leishmania mexicana. A metalloenzyme capable of protecting liposome-encapsulated proteins from phagolysosomal degradation by macrophages. J Biol Chem 1989; 264: 7483-9.

[95] Olobo JO, Anjili CO, Gicheru MM, et al. Vaccination of vervet monkeys against cutaneous leishmaniosis using recombinant Leishmania 'major surface glycoprotein' (gp63). Vet Parasitol 1995; 60: 199-212.

[96] Connell ND, Medina-Acosta E, McMaster WR, Bloom BR, Russell DG. Effective immunization against cutaneous leishmaniasis with recombinant bacille Calmette-Guérin expressing the Leishmania surface proteinase gp63. Proc Natl Acad Sci USA 1993; 90: 11473-

[97] Yang DM, Fairweather N, Button LL, McMaster WR, Kahl LP, Liew FY. Oral Salmonella typhimurium (AroA-) vaccine expressing a major leishmanial surface protein (gp63) preferentially induces $\mathrm{T}$ helper 1 cells and protective immunity against leishmaniasis. J Immunol 1990; 145: 2281-5.

[98] Gurunathan S, Klinman DM, Seder RA. DNA vaccines: immunology, application, and optimization. Ann Rev Immunol 2000; 18: 927-4.

[99] Valenzuela JG, Belkaid Y, Garfield MK, et al. Toward a defined anti-Leishmania vaccine targeting vector antigens. characterization of a protective salivary protein. J Exp Med 2001; 194: 331-42.

[100] Selvapandiyan A, Dey R, Nylen S, Duncan R, Sacks D, Nakhasi HL. Intracellular replication-deficient Leishmania donovani 
induces long lasting protective immunity against visceral leishmaniasis. J Immunol 2009; 183: 1813-20.

[101] Rivier D, Bovay P, Shah R, Didisheim S, Mauël J. Vaccination against Leishmania major in a CBA mouse model of infection. role of adjuvants and mechanism of protection. Parasite Immunol 1999; 21: 461-73.

[102] Cross GA. Antigenic variation in trypanosomes. secrets surface slowly. Bioessays 1996; 18: 283-91.

[103] Barry JD, McCulloch R. Antigenic variation in trypanosomes. enhanced phenotypic variation in a eukaryotic parasite $\mathrm{Adv}$ Parasitol 2001; 49: 1-70.

[104] Wilson RA, Coulson PS. Strategies for a schistosome vaccine. can we manipulate the immune response effectively? Microbes Infect 1999; $1: 535-43$.

[103] Hoffmann KF, James SL, Cheever AW, Wynn TA. Studies with double cytokine-deficient mice reveal that highly polarized Th1and Th2-type cytokine and antibody responses contribute equally to vaccine-induced immunity to Schistosoma mansoni. J Immunol 1999 15; 163: 927-38.

[105] Bungiro RD Jr, Greene J, Kruglov E, Cappello M. Mitigation of hookworm disease by immunization with soluble extracts of Ancylostoma ceylanicum. J Infect Dis 2001; 183: 1380-7.

[106] Tongren JE, Zavala F, Roos DS, Riley EM. Malaria vaccines. if at first you don't succeed. Trends Parasitol 2004; 20: 604-10.

[107] Dalton JP, Brindley PJ, Knox DP, et al. Helminth vaccines, from mining genomic information for vaccine targets to systems used for protein expression. Int J Parasitol 2003; 33: 621-40.

[108] Rinaldi G, Morales ME, Cancela M, Castillo E, Brindley PJ, Tort JF. Development of functional genomic tools in trematodes. RNA interference and luciferase reporter gene activity in Fasciola hepatica. PLoS Negl Trop Dis 2008; 2: e260.

[109] Bellofatto V, Palenchar JB. RNA interference as a genetic tool in trypanosomes. Methods Mol Biol 2008; 442: 83-94.

[110] Morales ME, Rinaldi G, Gobert GN, Kines KJ, Tort JF, Brindley PJ. RNA interference of Schistosoma mansoni cathepsin D, the apical enzyme of the hemoglobin proteolysis cascade. Mol Biochem Parasitol 2008; 157: 160-8.

[111] Knox DP, Geldhof P, Visser A, Britton C. RNA interference in parasitic nematodes of animals. a reality check? Trends Parasitol 2007; 23: 105-7.

[112] Delcroix M, Sajid M, Caffrey CR, et al. A multienzyme network functions in intestinal protein digestion by a platyhelminth parasite. J Biol Chem 2006; 281: 39316-29.

[113] Lightowlers MW, Rolfe R, Gauci CG. Taenia saginata. vaccination against cysticercosis in cattle with recombinant oncosphere antigens. Exp Parasitol 1996; 84: 330-8.

[114] Dalton JP, McGonigle S, Rolph TP, Andrews SJ. Induction of protective immunity in cattle against infection with Fasciola hepatica by vaccination with cathepsin $\mathrm{L}$ proteinases and with hemoglobin. Infect Immun 1996; 64: 5066-74.

[115] Willadsen P, Smith D, Cobon G, McKenna RV Comparative vaccination of cattle against Boophilus microplus with recombinant antigen Bm86 alone or in combination with recombinant Bm91. Parasite Immunol 1996; 18: 241-6.

[116] Mendez S, Zhan B, Goud G, et al. Effect of combining the larval antigens Ancylostoma secreted protein 2 (ASP-2) and metalloprotease 1 (MTP-1) in protecting hamsters against hookworm infection and disease caused by Ancylostoma ceylanicum. Vaccine 2005; 23: 3123-30.

[117] Méndez S, Belkaid Y, Seder RA, Sacks D. Optimization of DNA vaccination against cutaneous leishmaniasis. Vaccine 2002; 20: 3702-8.

[118] Doolan DL, Hoffman SL. Nucleic acid vaccines against malaria. Chem Immunol 2002; 80: 308-21.

[119] Abdulhaqq SA, Weiner DB. DNA vaccines. Developing new strategies to enhance immune responses. Immunol Res 2008; 42: 219-32.

[120] Redmond DL, Geldhof P, Knox DP. Evaluation of Caenorhabditis elegans glycoproteins as protective immunogens against Haemonchus contortus challenge in sheep. Int J Parasitol 2004; 34 : 1347-53.

[121] Haslam SM, Coles GC, Munn EA, Smith TS, Smith HF, Morris HR, Dell A. Haemonchus contortus glycoproteins contain N-linked oligosaccharides with novel highly fucosylated core structures. J Biol Chem 1996; 271: 30561-70.

[122] Britton C, Murray L. Using Caenorhabditis elegans for functional analysis of genes of parasitic nematodes. Int J Parasitol 2006; 36 651-9.

[123] Murray L, Geldhof P, Clark D, Knox DP, Britton C. Expression and purification of an active cysteine protease of Haemonchus contortus using Caenorhabditis elegans. Int J Parasitol 2007; 37: 1117-25.

[124] Geldhof P, Vercauteren I, Vercruysse J, Knox DP, Van Den Broeck W, Claerebout E. Validation of the protective Ostertagia ostertagi ES-thiol antigens with different adjuvantia. Parasite Immunol 2004; 26: 37-43.

[125] Hotez PJ, Zhan B, Bethony JM, et al. Progress in the development of a recombinant vaccine for human hookworm disease. the Human Hookworm Vaccine Initiative. Int J Parasitol 2003; 33: 1245-58.

[126] Graves PM. Comparison of the cost-effectiveness of vaccines and insecticide impregnation of mosquito nets for the prevention of malaria. Ann Trop Med Parasitol 1998; 92: 399-410.

[127] Knox DP. Redmond DL. Parasite-recent progress and problems associated with their development. Parasitology 2006; 133(Suppl 2): S1-8.

This is an open access article licensed under the terms of the Creative Commons Attribution Non-Commercial License (http://creativecommons.org/licenses/ by-nc/3.0/) which permits unrestricted, non-commercial use, distribution and reproduction in any medium, provided the work is properly cited. 